\title{
Characterization of dextrin-based hydrogels: Rheology, biocompatibility, and degradation
}

\author{
Joana Carvalho, ${ }^{1}$ Susana Moreira, ${ }^{1}$ João Maia, ${ }^{2}$ Francisco M. Gama ${ }^{1}$ \\ ${ }^{1} I B B$, Institute for Biotechnology and Bioengineering, Department of Biological Engineering, Universidade do Minho, \\ Campus de Gualtar, 4710-057 Braga, Portugal \\ ${ }^{2}$ I3N, Department of Polymer Engineering, Institute for Nanostructures, Nanomodelling and Nanofabrication, \\ Universidade do Minho, 4800-058 Guimarães, Portugal
}

Received 1 October 2008; revised 26 March 2009; accepted 7 April 2009

Published online 30 June 2009 in Wiley InterScience (www.interscience.wiley.com). DOI: 10.1002/jbm.a.32553

\begin{abstract}
A new class of degradable dextrin-based hydrogels (dextrin-HEMA) was developed. The hydroxyethyl methacrylate ester (HEMA) hydroxyl groups were activated with $N, N^{\prime}$-carbonyldiimidazole (CDI), followed by their coupling to dextrin, yielding a derivatized material that can be polymerized in aqueous solution to form hydrogels. A comparative study of the stability of the dextrin-HEMA hydrogels and dextrin-vinyl acrylate (dextrin$\mathrm{VA}$, produced in previous work) revealed that only the firsts are effectively hydrolyzed under physiological conditions. A severe mass loss of dextrin-HEMA gels occurs over time, culminating in the complete dissolution of the gels. Rheologic analysis confirmed that physical structur-
\end{abstract}

ing is less pronounced when dextrin is modified with methacrylate side groups. The biocompatibility results revealed that the dextrin hydrogels have negligible cell toxicity, irrespective of the hydrogel type (HEMA and VA), allowing cell adhesion and proliferation. Gathering the biocompatibility and the ability to tailor the release profiles, we consider dextrin a promising biomaterial for biomedical applications, namely for controlled release. (C) 2009 Wiley Periodicals, Inc. J Biomed Mater Res 93A: 389-399, 2010

Key words: dextrin; hydrogels; biocompatibility; degradation kinetics

\section{INTRODUCTION}

Hydrogels are a class of three-dimensional, hydrophilic polymeric networks that can absorb substantial amounts of water. In general, hydrogels have a good biocompatibility and are capable of resembling natural tissues, allowing for a favorable controlled interaction with living systems. ${ }^{1}$ For this reason, these materials have found widespread application in the biomedical, biotechnological, and pharmaceutical fields. ${ }^{2-8}$ Hydrogels can be used in tissue engineering, as scaffolds to support and promote tissue regeneration and also as attractive systems for the controlled release of pharmaceutically active molecules. $^{9-14}$

An important class of hydrogels is obtained by the polymerization of acrylate esters [e.g., vinyl acrylate, methacrylate, hydroxyethyl methacrylate] through the polymerization of the water-soluble functional-

Correspondence to: F. M. Gama; e-mail: fmgama@deb. uminho.pt

(C) 2009 Wiley Periodicals, Inc. ized polymers. ${ }^{15-21}$ In previous work, we have described a new dextrin-based hydrogel in which the polysaccharide is functionalized with VA. The reactive double bonds cross-links by free-radical polymerization in aqueous solution. ${ }^{22}$ The degradation of the ester bonds present in the crosslinks of those polymeric networks is very slow under physiological conditions. However, the hydrogels could be rendered degradable through the incorporation of an enzyme, which prove to be an effective route to modulate the gel degradation and the release of entrapped molecules. Alternatively, the functionalization of the polymer with hydroxyethyl methacrylate ester (HEMA) was attempted, as already described for dextran, as an efficient approach to obtain hydrogels with desirable spontaneous (nonenzymatic) degradation kinetics. ${ }^{16-19,23}$ The production of dextrin-HEMA hydrogels is reported in this work. It should be remarked that dextrin, an $\alpha-(1 \rightarrow 4)$ glucose polymer, was used instead of dextran $[\alpha-$ $(1 \rightarrow 6)]$. A comparative study of the stability of the two different acrylated dextrins (dextrin-VA and dextrin-HEMA) was also carried out. The rheologic analysis allowed exploring the time evolution of the 
viscoelastic behavior of the hydrogels, providing a qualitative characterization of the network, as well as the structural changes occurring during degradation. The biocompatibility of the hydrogels is an essential issue regarding their pharmaceutical and biomedical applicability. ${ }^{2,24-28}$ In this context, in vitro toxicity of the hydrogels was evaluated (following the guidelines from ISO10993) and observations with both fluorescence and phase-contrast inverted light microscopy were also carried out as indicators of cell viability and morphology.

\section{MATERIALS AND METHODS}

\section{Materials}

Dextrin-Koldex 60 starch was a generous gift from Tate \& Lyle (Decatur, IL). VA was from Aldrich, $N, N, N^{\prime}, N^{\prime}-$ tetramethylenethylenediamine (TEMED) and ammonium persulfate (APS) were purchased from BioRad, dimethylsulfoxide (DMSO) and acetone were from AppliChem. 2-Hydroxyethyl methacrylate (HEMA), hydroquinone monomethyl ether (>98\%), 4-(N,N-dimethylamino)pyridine (DMAP, 99\%), $N, N^{\prime}$-carbonyldiimidazole (CDI, 98\%), and tetrahydrofuran (THF) were from Fluka. Dulbecco's modified Eagle medium (DMEM) with $4500 \mathrm{mg}$ glucose/liter, L-glutamine, $110 \mathrm{mg}$ sodium pyruvate/liter and $\mathrm{NaHCO}_{3}$ (Sigma) was supplemented with $10 \%$ calf bovine serum (CBS) (Gibco, UK) and 1\% of a penicillin/streptomycin solution (Sigma). This medium is further referred to as DMEM complete medium. MTT (3-[4,5-dimethylthiazol-2yl]-2,5-diphenyl-tetrazolium bromide) was obtained from Sigma and transwell plates were purchased from Orange Scientific (Belgium). For the fluorescence microscopy assays, the LIVE/DEAD ${ }^{\circledR}$ Viability/Cytotoxicity Kit for mammalian cells, from Molecular Probes (USA), was used. Regenerated cellulose tubular membranes with 3500 molecular weight cutoff (MWCO) were obtained from Membrane Filtration Products (Seguin, USA). All other chemicals and solvents used in this work were of the highest purity commercially available.

\section{Synthesis of HEMA-imidazolyl carbamate (HEMA-CI)}

The synthesis of HEMA-CI was performed as described by vanDijkWolthuis et al. ${ }^{18} \mathrm{CDI}(1.62 \mathrm{~g}, 10 \mathrm{mmol})$ was dissolved in about $15 \mathrm{~mL}$ anhydrous THF in a nitrogen atmosphere and HEMA (1.30 g, $10 \mathrm{mmol})$ was added. The reaction mixture was stirred for $16 \mathrm{~h}$ at ambient temperature. After addition of a small amount of hydroquinone monomethyl ether $(60 \mathrm{mg})$, the solvent was evaporated and the crude product was dissolved in ethyl acetate, extracted with water and dried on anhydrous $\mathrm{MgSO}_{4}$. After filtration, hydroquinone monomethyl ether $(60 \mathrm{mg})$ was added once more and the solvent evaporated.

\section{Synthesis of dextrin-HEMA}

The modification of dextrin with HEMA was performed as described by vanDijkWolthuis et al. ${ }^{18}$ with few modifications. Briefly dextrin (10.0 g) was dissolved in dried DMSO $(90 \mathrm{~mL})$ in a nitrogen atmosphere. After dissolution of DMAP (2.0 g), HEMA-CI was added, in the molar ratios of 0.25 and 0.50 of HEMA-CI to glucopyranose residues in dextrin. The solution was stirred at room temperature for 4 days, after which the reaction was stopped by adding 2 $\mathrm{mL}$ of concentrated $\mathrm{HCl}$. The reaction mixture was dialyzed for 2-3 days against demineralized water at $4{ }^{\circ} \mathrm{C}$. The methacrylated dextrin was lyophilized and the final white fluffy product was stored at $-20^{\circ} \mathrm{C}$ until use. The degree of acrylate substitution (DS) of the methacrylated dextrin was determined by proton nuclear resonance spectroscopy $\left({ }^{1} \mathrm{H}\right.$ NMR) in $\mathrm{D}_{2} \mathrm{O}$. Briefly, ${ }^{1} \mathrm{H}$ NMR spectra were recorded in $\mathrm{D}_{2} \mathrm{O}(10 \mathrm{mg}$ in $1 \mathrm{~mL})$ and $\mathrm{DS}$ was calculated using Eq. (1):

$$
\mathrm{DS} \%=\frac{x}{y} \times 100
$$

in which $x$, is the average integral of the protons at the double bond ( $\delta$ at 5.8 and $6.2 \mathrm{ppm}$ ) and $y$ is the integral of the anomeric proton ( $\delta$ around $5.4 \mathrm{ppm}$ ).

\section{General procedure for the preparation of dextrin-VA and dextrin-HEMA hydrogels}

Dextrin-VA monomers were synthesized from dextrin in DMSO in the presence of different amounts of VA and the degree of acrylate substitution (DS) was determined by proton nuclear resonance spectroscopy $\left({ }^{1} \mathrm{H}\right.$ NMR) in $\mathrm{D}_{2} \mathrm{O}$ as previously described. ${ }^{22}$ The hydrogel slabs with different DS (20 and $40 \%$ ) were prepared by radical polymerization of aqueous solution of either dextrin-VA or dextrinHEMA (300 mg/mL). The gelation reactions were initiated by adding $90 \mu \mathrm{L}$ APS ( $80 \mathrm{mg} / \mathrm{mL}$ in phosphate buffer) and $90 \mu \mathrm{L}$ TEMED $(13.6 \%(v / v)$ in water, $\mathrm{pH}$ adjusted to 8.0 with $\mathrm{HCl}$ ) and allowed to occur for $30 \mathrm{~min}$ at room temperature.

\section{Standard degradation conditions}

Two sets of hydrogels were used. After the polymerization reaction, gels were removed from the casting recipients. One set of hydrogels was immediately lyophilized to determine the initial dry weight $\left(W_{\mathrm{i}}\right)$. The other one was immersed in $50 \mathrm{~mL}$ of phosphate buffer saline (PBS) $\mathrm{pH}$ 7.4 and kept in a thermostated room at $37^{\circ} \mathrm{C}$. At time intervals, gels were removed, blotted with filter paper to remove water excess, weighted $\left(W_{\mathrm{s}}\right.$, weight in the swollen state) and lyophilized to determine the dry weight, $W_{\mathrm{d}}$. The swelling ratio (SR) and the mass loss ( $\left.m_{\text {loss }}\right)$ were calculated according to Eqs. (2) and (3), respectively:

$$
\mathrm{SR}=\frac{W_{\mathrm{s}}-W_{\mathrm{d}}}{W_{\mathrm{d}}}
$$




$$
m_{\text {loss }}=100-\left(\frac{W_{\mathrm{i}}}{W_{\mathrm{d}}} \times 100\right)
$$

The hydrogel degradation time is defined as the time needed for complete degradation $(W=0)$.

\section{Rheologic measurements}

The rheologic measurements were performed in a controlled-stress Rheologica rheometer at a controlled temperature $\left(25^{\circ} \mathrm{C}\right)$. A plate-plate measuring system was used $(\varnothing$ $25 \mathrm{~mm}$ ) and the gap between the plates was $1 \mathrm{~mm}$. For the experiments, polymerized hydrogels with 25-mm diameter and 1-mm thickness, were immersed in $50 \mathrm{~mL}$ of PBS at $\mathrm{pH} 7.4$ and kept in a thermostated room at $37^{\circ} \mathrm{C}$ to allow degradation, with the exception of the initial time measurements $(t=0)$, in which hydrogels were used immediately after polymerization. At set time intervals, gels were removed from the containers and placed between the plates of the measuring system. Two sets of experiments were performed:

(a) Stress sweep experiments were carried out to optimize the applied stress used in the frequency-oscillation experiments. The lower limit of the applied stress is determined by the sensitivity of the instrument. On the other hand, the upper limit of the applied stress will be influenced by the linear viscoelastic range and the presence of slip effects.

(b) Once the range of linear viscoelastic response was determined, experiments were performed in Small Amplitude Oscillatory Shear, SAOS, at a fixed frequency of $1 \mathrm{~Hz}$ and a stress known to yield a good quality signal in the linear viscoelastic regime.

\section{Cell culture}

Mouse embryo fibroblasts 3T3 (ATCC CCL-164) were grown in DMEM complete medium, at $37^{\circ} \mathrm{C}$, in a fully humidified air containing $5 \% \mathrm{CO}_{2}$ (IR auto Flow). The cells were fed every 2-3 days. When cells reached confluence, the culture medium was discarded and the cells were detached with $2 \mathrm{~mL}$ of $0.25 \%(w / v)$ trypsin-EDTA [1:250, from porcine pancreas (Sigma)] solution for $15 \mathrm{~min}$ at $37^{\circ} \mathrm{C}$, and $6 \mathrm{~mL}$ of DMEM complete medium was added to inactivate the trypsin after cell detachment. The cells were then centrifuged (10 $\mathrm{min}, 1000 \mathrm{rpm})$ and resuspended in culture medium before use.

\section{Mitochondrial metabolic activity assay}

MTT solution $(0.45 \mathrm{mg} / \mathrm{mL}$ in DMEM complete medium without phenol red) in an amount equal to $10 \%$ of the culture volume was added to each well. After $3 \mathrm{~h}$ of incubation at $37^{\circ} \mathrm{C}$, the MTT solution was removed and the insoluble formazan crystals formed in the bottom of the wells were dissolved in a dimethylsulfoxide volume equal to the original culture volume. ${ }^{29}$ The absorbance was measured at $570 \mathrm{~nm}$ using a plate reader (SynergyHT, BioTek). The CPII was calculated using Eq. (4):

$$
\mathrm{CPII}=100-\left(\frac{\mathrm{DO}_{570} \text { of test culture }}{\mathrm{DO}_{570} \text { of control culture }} \times 100\right)
$$

\section{Direct contact assay}

A fibroblast suspension $(3 \mathrm{~mL})$ containing $3 \times 10^{4}$ cells / $\mathrm{mL}$ was plated into each well of a six-well plate, to yield a final density of $10^{4}$ cells $/ \mathrm{cm}^{2}$. After reaching a state of subconfluence (after $24 \mathrm{~h}$ ), hydrogel discs (Ø $4 \mathrm{~mm}, 2-\mathrm{mm}$ thickness) were placed on the wells, in direct contact with cells. At regular time intervals, the cell morphology and viability was assessed with the Live/Dead fluorescence labeling. Positive and negative controls (discs of latex and agar gel) were also used.

\section{Adhesion assay}

The cell adhesion in dextrin-VA hydrogels was evaluated. After $2 \mathrm{~h}$ of polymerization, the hydrogels formed at the bottom of the wells were washed, first with PBS and secondly with DMEM complete medium $(3 \times 200 \mu \mathrm{L})$; then $100 \mu \mathrm{L}$ of a fibroblast suspension containing $3 \times 10^{4}$ cells $/ \mathrm{mL}$ was plated into each well, to yield a final density of $10^{4}$ cells $/ \mathrm{cm}^{2}$. The culture medium was refreshed $(100$ $\mu \mathrm{L}$ ) every 2 days. For the control assays, cells were grown directly in the bottom of the wells. At regular time intervals, the hydrogels were washed with PBS $(3 \times 100 \mu \mathrm{L})$ to remove floating cells, and the cell layer was trypsinized before conducting the colorimetric (MTT) and counting assays, an approach that resulted in more accurate and reliable results.

\section{Microscopy assays}

At regular time intervals, cell growth and morphology were evaluated by observation with a phase-contrast inverted light microscope. Cytotoxic effects were ranked in cellular death, cell adhesion ability, and induction of morphological changes. Additionally, cells were labeled with LIVE/DEAD Viability/Cytotoxicity Kit and observed by fluorescence microscopy.

\section{RESULTS AND DISCUSSION}

In previous work, we have demonstrated the production of a new dextrin hydrogel, through the transesterification of the polymer with VA, followed by free-radical polymerization. ${ }^{22}$ In that work, it was demonstrated that the esterification occurs on the 2(preferentially) and 3-hydroxyl groups in the glucopyranose ring. Furthermore, a comprehensive study was performed, by solid state NMR, allowing the detection of adsorbed, linked and polymerized 


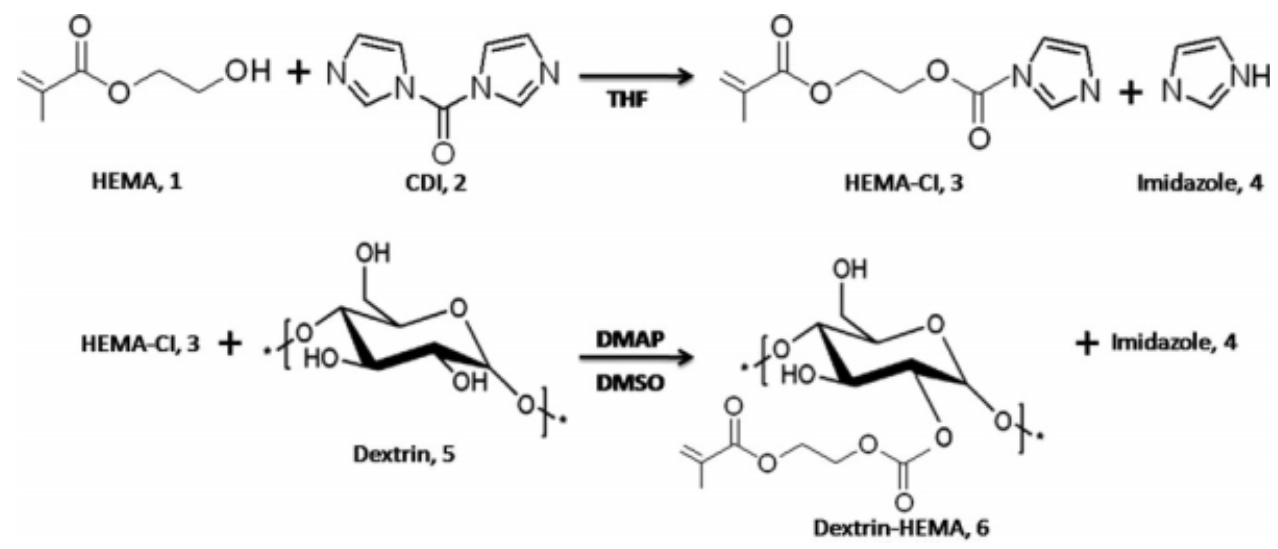

Figure 1. Synthesis of dextrin-HEMA.

acrylic molecules. The dextrin-VA hydrogel degrades slowly and to a limited extent, under physiological conditions, which is a limitation in light of their application as controlled delivery systems or in tissue engineering. Several studies reported the successful functionalization of dextran with a methacrylate ester (HEMA) and it was shown that hydrogels obtained by polymerization of this compound, are able to degrade in desirable timeframes. ${ }^{16-19,23}$ In this work, a similar dextrin modification was attempted using the same chemistry. As dextrin is rather distinct from dextran (different size, different structure), distinct properties are expected and characterized in this work.

\section{Activation of HEMA with CDI and synthesis of dextrin-HEMA}

As previously described for dextran, ${ }^{18}$ it was also possible to couple HEMA to the hydroxyl groups of dextrin via a mixed carbonate ester. This linkage can be effectively introduced by using $N, N^{\prime}-\mathrm{CDI}$ as an intermediate activation step. In this activation reaction, HEMA reacts with an equimolar amount of $\mathrm{CDI}$, in THF, to produce HEMA-imidazolyl carbamate, hereafter referred to as HEMA-CI (Fig. 1). In the next step, HEMA-CI reacts with dextrin, using DMSO as reaction medium. ${ }^{1} \mathrm{H}$ RMN analysis shows that dextrin-HEMA with different DS (ranging from ca. 10 to $40 \%$ ) can be obtained, by varying the molar ratio of HEMA-CI to dextrin (Fig. 2). It is assumed that the dextrin esterification with HEMA occurs preferentially at the 2- and 3-hydroxyl groups of the glucopyranose ring, as previously shown for dextrinVA. In addition, the modified polymer was successfully recovered by lyophilization, after dialysis.

\section{Swelling and degradation behavior of dextrin-VA and dextrin-HEMA hydrogels}

On exposure to an aqueous solution, the water molecules are drawn into the hydrogel network. The

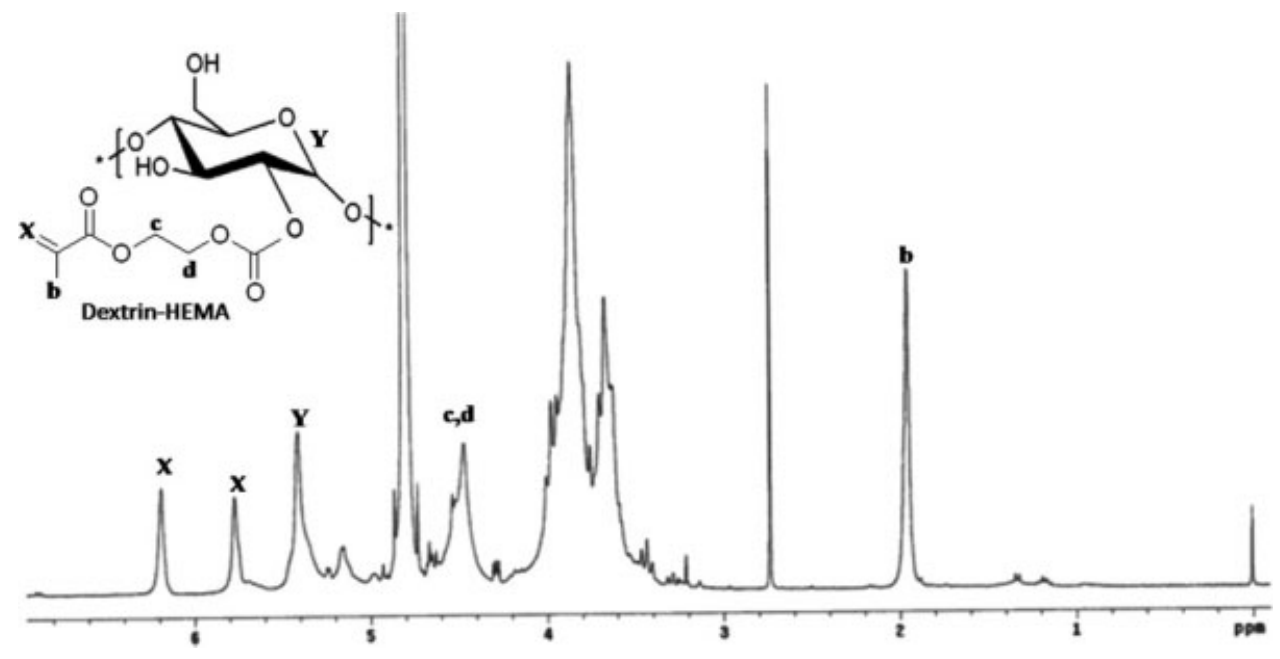

Figure 2. Dextrin-HEMA ${ }^{1} \mathrm{H}$ NMR spectrum. 

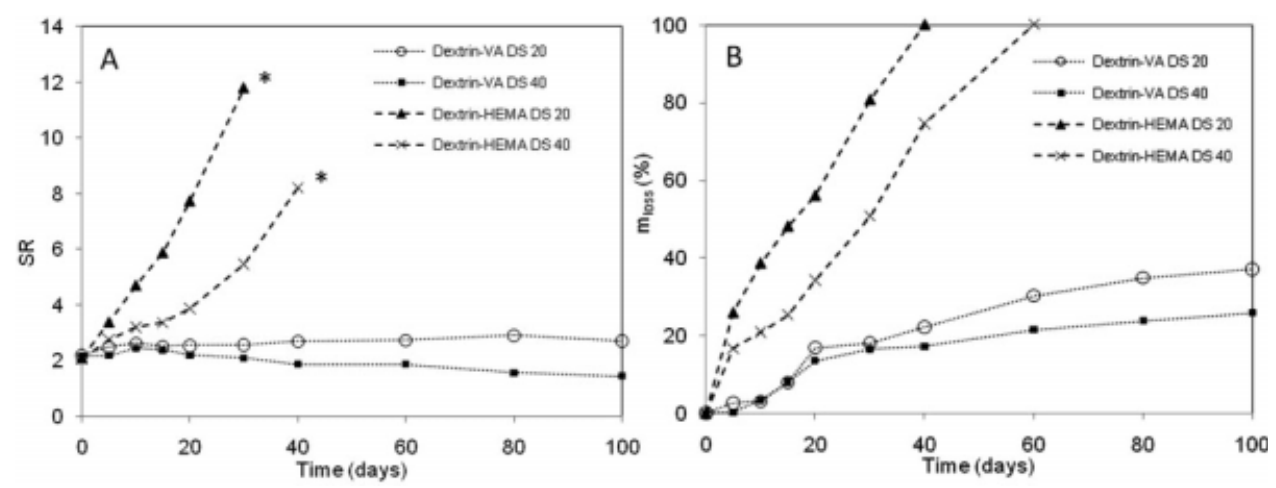

Figure 3. Degradability of dextrin-based hydrogels. Swelling ratio (A) and mass loss as a function of time, when immersed in PBS pH 7.4, at $37^{\circ} \mathrm{C}$ (data representative of three experiments; the standard deviation was always inferior to $5 \%$ ). ( ${ }^{*}$ From day 40 the hydrogel structure was severely damaged, preventing the SR measurements).

initial and relaxed state of the hydrogel (dry state) is, thereby, replaced by a swollen state. As the water molecules are going to occupy some space, the meshes of the network will start expanding, allowing for the uptake of more water molecules. The stretching caused by the swelling process is counterbalanced by the covalent or physical crosslinking of the network, preventing its destruction and creating an equilibrium swelling state. ${ }^{30}$ The functionality and number of linkages in the hydrogel, as well as the monomer concentration, influence not only the SR, but also the erosion profile and the overall time for degradation. ${ }^{5}$ In this work, a comparative study on the swelling and degradation behavior, was performed in hydrogels with two distinct ester functions [acrylate (dextrin-VA) and methacrylate (dextrin-HEMA)], and the influence of the DS on these parameters was also evaluated. Dextrin-VA hydrogels with DS 20 or DS 40 were compared with dextrin-HEMA with the same DS.

As can be seen in Figure 3(A), for dextrin-VA, at physiological $\mathrm{pH}$, in the initial period of the incubation (around 5 days), a very slight increase in the SR is detected for both degrees of substitution (DS 20 or DS 40). Nevertheless, neither gel showed a significant increase in swelling over 100 days, indicating that no significant hydrolysis of the polymerized acrylate esters occurred. As expected, greater swelling values (in the range 2.5-3) were found for the DS 20 networks, compared with those (in the range 1.62.1) obtained with the higher DS, because in highly crosslinked hydrogels the mobility of the polymer chains is hindered, lowering the ability to swell.

Contrarily to the dextrin-VA hydrogels, in which the SR remains almost constant during the time course of the experiment, in the case of dextrinHEMA, a progressive swelling occurred over the time, until the complete dissolution of the hydrogels is accomplished. The increased swelling is likely a consequence of the hydrolysis of the methacrylate bonds in the crosslinked structure. The cleavage of the ester bonds leads to a decrease in the crosslinking density, allowing for the network to expand and swell, absorbing more water. As can be seen in Figure 3(A), the SR values reach a maximum at the time that the gels starts to dissolve and dextrin hydrogels most likely bear few crosslinks per polymer chain. The mass loss of dextrin-VA/HEMA hydrogels with different DS (DS 20 and DS 40) was also monitored as a function of time. Figure 3(B) summarizes the degradation times of all dextrin gels investigated. This figure shows that for both gel systems (dextrin-VA or dextrin-HEMA), the degradation time increased with an increasing DS. It seems obvious that an increasing DS at a fixed initial water content (all the hydrogels have the same initial water content of about $70 \%$ ), results in a network with a higher crosslinking density. In these cases, to accomplish the network dissolution, more cross-links have to be hydrolyzed, which requires more time. When comparing the two hydrogels systems, a linear mass loss profile can be observed in dextrin-VA, until day 20 , with a rate of roughly $0.5 \%$ of gel solubilized per day. However, following this initial period, the rate slows down, and at day 100, only about 40 and $25 \%$ of mass loss is detected for DS 20 and DS 40 hydrogels, respectively. Probably, the mass losses observed correspond to the release of nonpolymerized material.

Contrarily, as shown in Figure 3(B), for dextrinHEMA hydrogels, the mass loss was dramatic, reaching about $30 \%$ for DS 20 and $50 \%$ for DS 40 , after 20 days of incubation. In this case, hydrogels start to disintegrate quickly, until a complete dissolution is achieved. As also expected, while for a DS 20 dextrin-HEMA hydrogel, the dissolution took place in $\sim 35$ days, in case of DS 40 hydrogels, further time was needed to accomplish dissolution (60 days). These results suggest that, in the case of dextrin-HEMA hydrogels, the degradation occurs via a 

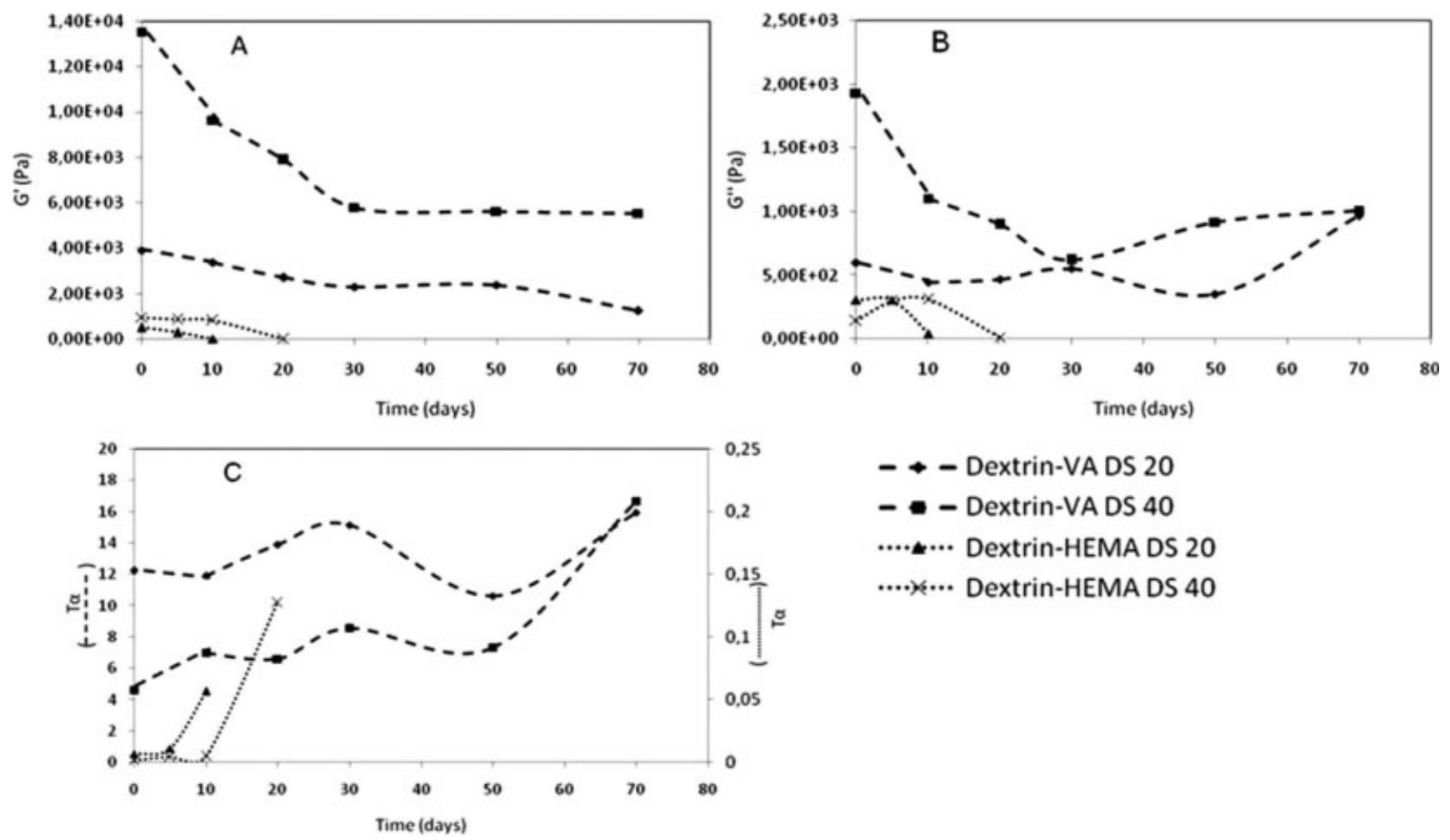

Figure 4. Rheologic measurements of the different hydrogel samples. Frequency is $1 \mathrm{~Hz}$ and stress varies to keep the response in the linear viscoelastic regime. Representative results selected among several assays are shown.

homogeneous bulk erosion process. In this case, the SR would be expected to increase with the incubation time, because of the loosened polymer network because, although hydrolyzed, the polymer chains remain in the matrix, allowing the network to become looser and to continuously swell, until the dissolution is complete. ${ }^{31-34}$ In the case of dextrinVA hydrogels, it seems that the initial mass loss can be attributed to the release of a fraction of unbound molecules which were physically entrapped in the matrix at the time of polymerization. As diffusion occurs, the small molecules are released out of the network. A stabilization of the swelling values is, thus, observed at the time that all the unbound molecules are released. In contrary to dextrin-HEMA, which is hydrolytically labile, dextrin-VA hydrogel is very stable under physiological conditions.

\section{Mechanical properties}

Mechanical properties of hydrogels are very important for pharmaceutical applications. For example, the integrity of a drug delivery device during the lifetime of the application is crucial. The system must protect a sensitive therapeutic agent, such as protein, maintaining its integrity until it is released out of the system. Changes in the crosslinking den- sity of the hydrogels have been routinely applied to achieve the desired mechanical properties. Increasing the crosslinking will result in a stronger gel with a more brittle structure, which sometimes is not desirable because it shall interfere with the release profiles. Hence, a compromise must be achieved between a relatively strong but yet elastic structure. $^{35}$ It is well established that the release of the proteins from a hydrogel is clearly influenced by its degradation rates. ${ }^{36}$ Dynamic mechanical analysis, performed on the hydrogel, provide quantitative information on the viscoelastic and rheologic properties of the material, by measuring the mechanical response of the samples as they are deformed. The elastic (also real or storage) modulus $G^{\prime}$ and the viscous (also imaginary or loss) modulus $G^{\prime \prime}$ are presented. The storage modulus [G', Fig. 4(A)] of a hydrogel is proportional to the amount of elastic chains within the polymer network. ${ }^{37}$ Figure 4 shows the rheologic results for all the hydrogels and it is clear that the results are in good agreement with the degradability studies above. Dextrin-HEMA hydrogels show lower $G^{\prime}$ values, as compared with the dextrin-VA ones, indicating that physical structuring is less pronounced when dextrin is modified with methacrylate side groups. Overall, it is possible to broadly correlate an increase in SR with a decrease in both the elastic and viscous responses, $G^{\prime}$ and $G^{\prime \prime}$ 
respectively, because the gels become softer as they swell, which tends to decrease $G^{\prime}$, whereas the increased water absorption, because of the hydrolysis of the methacrylate bonds in the crosslinked structure, leads to a decrease in $G^{\prime \prime}$ [Fig. 4(B)].

In the case of the dextrin-VA gels, $G^{\prime}$ and $G^{\prime \prime}$ decrease for short incubation times and stabilize after $\sim 30$ days. As there is a large uptake of water and these gels do not degrade, the viscous response increases slightly relatively to the elastic one, as reflected by the slight increase in $\tan (\alpha)$, but the viscoelastic behavior clearly remains that of a soft solid [Fig. 4(C)]. The DS has an appreciable impact on the storage modulus. Dextrin-VA hydrogels with a higher DS (DS 40) has more crosslink sites, which results in a denser mesh structure, more network chains, and an associated higher $G^{\prime}$, compared with the lower DS (DS 20). In the case of the dextrinHEMA gels, the degradation is very severe, with the viscoelastic response quickly approaching that of a inelastic low-viscosity liquid, that is, low $G^{\prime}$ and $G^{\prime \prime}$ and $\tan (\alpha) \gg 1$. This degradation culminated in the impossibility to perform any tests on the dextrinHEMA gels, because of their extreme fragility above 20 days of rest.

From these results, it could be concluded that a good correlation exists between the influence of the ester function (methacrylate/acrylate) used in the polymer derivatization, as well as the grafted group percentage (DS), on the rheologic degradation rates.

\section{In vitro biocompatibility assessment}

To evaluate the cytotoxicity of the two acrylatederivatized dextrins, we have studied the effects of these materials in a mouse embryo fibroblast culture, namely in the cell proliferation and adhesion on the hydrogel, morphological features, and cell death.

\section{Extraction assay}

As previously described, on exposure to aqueous solutions, hydrogels can release leachable products and, therefore, the cytotoxicity of hydrogel extracts must be evaluated. For that purpose, fibroblast cultures were exposed to extracts of hydrogels, prepared using dextrin-VA and dextrin-HEMA with different DS (20 and 40\%). Viability was quantified using the MTT assay. The results are shown in Table I. The extracts induced a reduction inferior to $15 \%$ in the mitochondrial metabolic activity of fibroblasts, as compared with the control. No significant differences were found between the two classes of hydrogels (HEMA and VA). The DS of the hydrogel network does also not produce major differences in the CPII.
TABLE I

Cell Proliferation Inhibition Index of Mouse Embryo Fibroblasts, Cultured in the Presence of Extracts Obtained from Dextrin Hydrogels

\begin{tabular}{lcc}
\hline \multirow{2}{*}{ Hydrogel } & \multicolumn{2}{c}{ CPII $(\%)^{\mathrm{a}}$, DS $(\%)^{\mathrm{b}}$} \\
\cline { 2 - 3 } & 20 & 40 \\
\hline Dextrin-VA & $14.9 \pm 1.6$ & $14.8 \pm 2.1$ \\
Dextrin-HEMA & $14.2 \pm 1.3$ & $13.7 \pm 1.7$
\end{tabular}

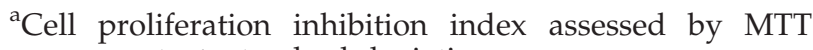
measurements \pm standard deviation.

${ }^{b}$ Degree of substitution defined as the amount of acrylate groups per 100 glucopyranose residues, determined by liquid NMR spectroscopy.

\section{Direct contact}

The cytotoxicity of dextrin-VA and dextrin-HEMA hydrogels was also assessed using a direct contact method. For that purpose, hydrogel discs as well as a positive and negative control (latex rubber and agar, respectively) were used. Each sample was placed on top of a subconfluent cellular layer on a six-well plate, as described in the material and methods section. Figure 5 shows the results obtained with the fluorescence labeling of the cell layers.

As can be seen, the latex rubber is, as expected, highly cytotoxic. Some cells were floating in the medium and the ones that remained attached are dead as well (red labeled). Differences in the morphology were found in the cells underneath the hydrogels. These cells appeared clustered, when compared with the control cultures, but all of them remained alive, as shown by the green fluorescence (data not shown). The cell proliferation under the dextrin hydrogel was lower than the one observed in the control plate. Massia and Stark, ${ }^{38}$ and more recently Ferreira et al. ${ }^{39,40}$ using human skin fibroblasts, have already reported this effect which is related to mechanical compression of cells or poor oxygenation and nutrient diffusion, because of the physical presence of the network. The cells in contact with the negative control (agar) and the dextrin hydrogels, irrespective of the chemistry and DS, exhibited similar morphology and proliferation rates, thus, the hydrogels can be considered nontoxic.

\section{Cell adhesion and proliferation on dextrin hydrogels}

The cell adhesion on the hydrogel surfaces was evaluated using samples with different DS (20 and $40 \%$ ). The results were expressed as a percentage of the adhesion on cell culture polystyrene plates (TCPP), estimated by cell counting. Additionally, the 

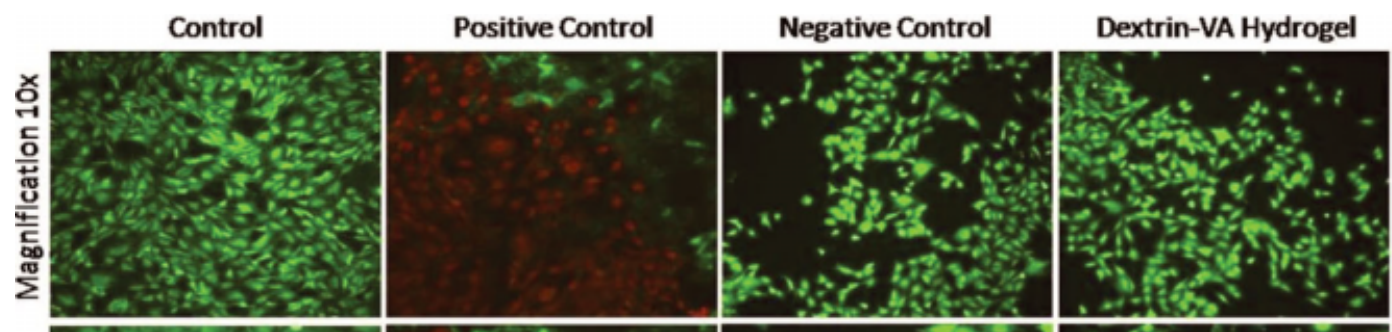

Dextrin-HEMA Hydrogel
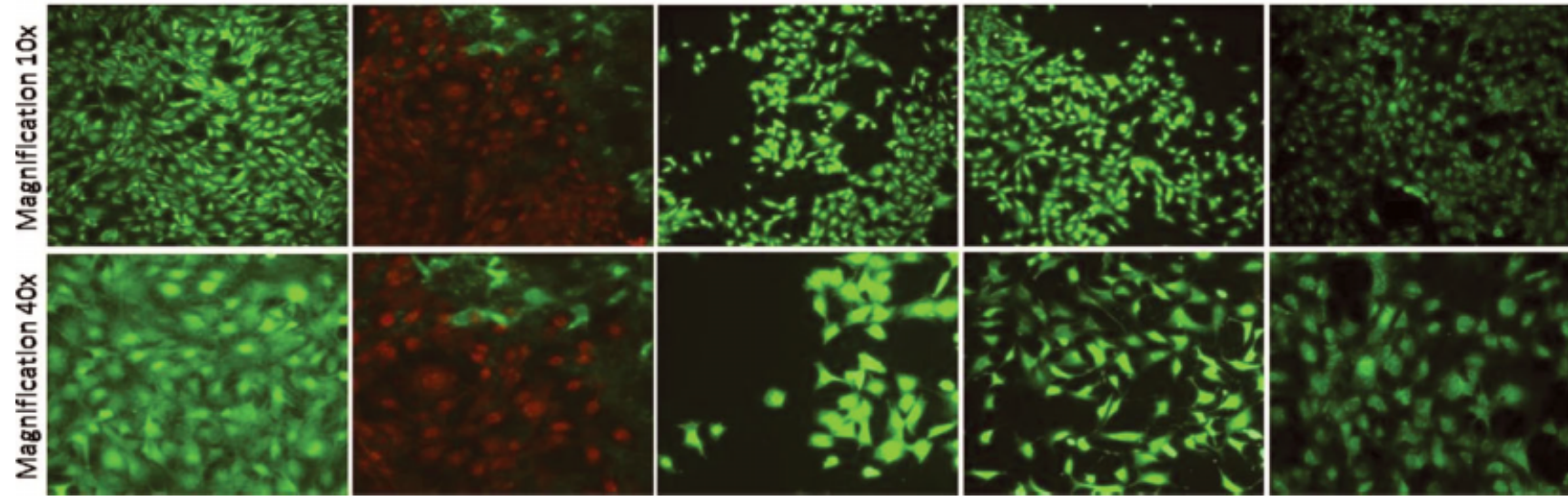

Figure 5. Fluorescence labeling of mouse embryo fibroblasts. Micrographs show the control culture, the cell layers in contact with the positive (latex rubber) and negative (agar) controls, and DS 20 dextrin hydrogels (VA/HEMA), after 24 h of incubation (magnification $\times 10$ and $\times 40$ ). [Color figure can be viewed in the online issue, which is available at www. interscience.wiley.com.]

mitochondrial activity of the adherent cells was also assessed in a parallel experiment. This study showed that the hydrogels, once again irrespective of the chemistry (HEMA or VA) and DS, are as expected, less adhesive than TCPP. The resistance of the hydrogel surfaces to cell adhesion is, as described
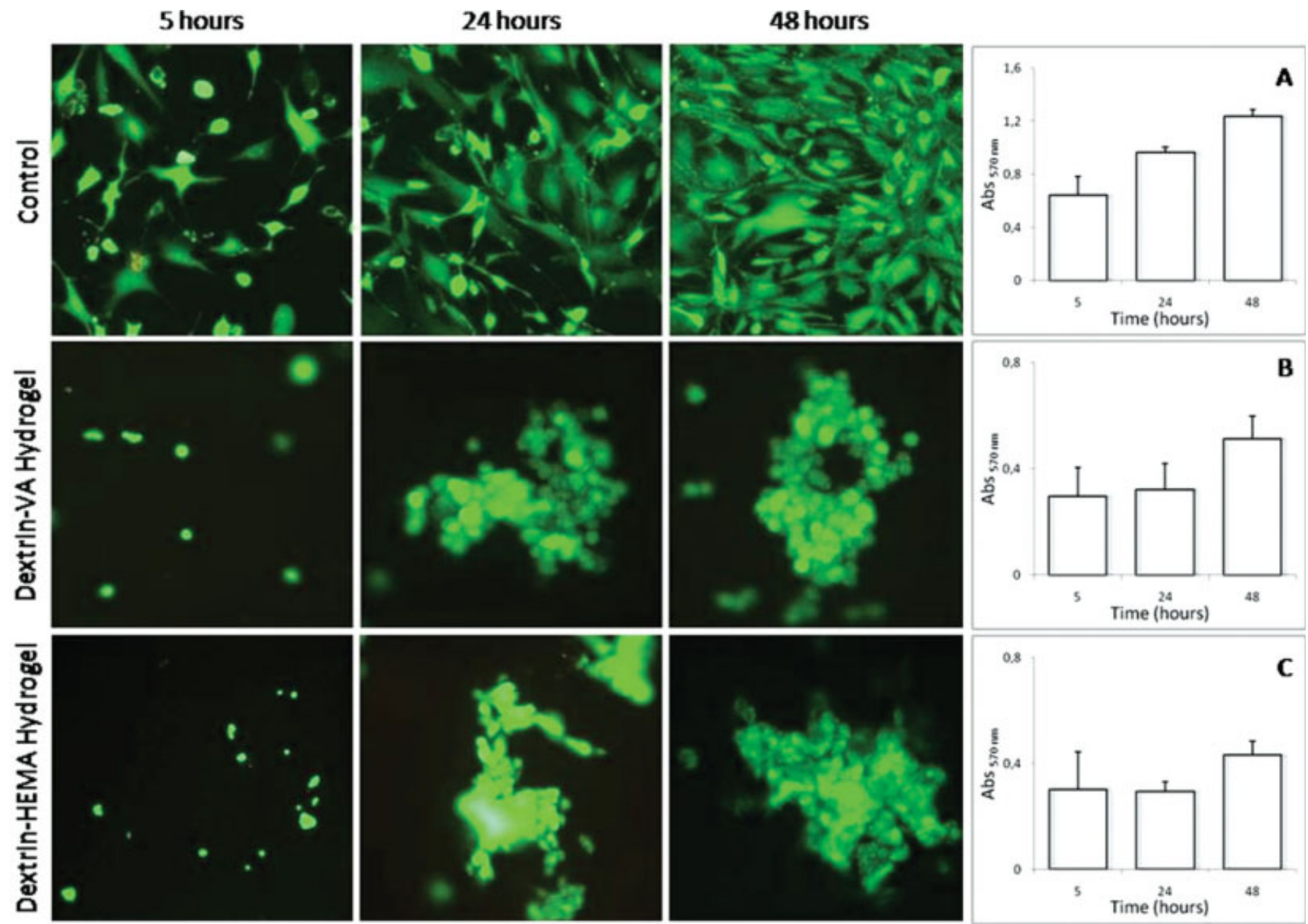

Figure 6. Fluorescence labeling of mouse embryo fibroblasts. Micrographs show control culture and cells seeded on the surface of DS 20 dextrin hydrogels (VA/HEMA), after 5, 24, and $48 \mathrm{~h}$ of incubation (magnification $\times 10$ ). A-C: Graphics correspond to the MTT absorbance values at $570 \mathrm{~nm}$ through time course of the assay. [Color figure can be viewed in the online issue, which is available at www.interscience.wiley.com.] 
TABLE II

Mouse Embryo Fibroblasts Adhesion onto Dextrin-VA and Dextrin-HEMA Hydrogels with Different DS Values (\% Estimated Taking the Cell Growth on TCPP as Reference)

\begin{tabular}{lccc}
\hline \multirow{2}{*}{ Hydrogel } & & \multicolumn{2}{c}{ Adhesion (\%) } \\
\cline { 3 - 4 } & DS (\%) & After $1 \mathrm{~h}$ & After $5 \mathrm{~h}$ \\
\hline Dextrin-VA & 20 & $23.4 \pm 2.0$ & $56.5 \pm 4.4$ \\
& 40 & $22.8 \pm 3.8$ & $59.1 \pm 2.7$ \\
Dextrin-HEMA & 20 & $25.7 \pm 4.6$ & $59.2 \pm 3.7$ \\
& 40 & $23.9 \pm 3.1$ & $59.7 \pm 2.6$ \\
\hline
\end{tabular}

for other polysaccharides such as dextran, presumably because of poor protein adsorption onto the hydrophilic and nonionic polymer. ${ }^{41-43}$ Indeed, cellular adhesion on hydrophilic materials such as hyaluronic acid and PEG-based hydrogels was reported to be extremely sparse. ${ }^{44,45}$ In other cases, as recently described in pullulan hydrogels using smooth muscle cells, cellular adhesion, and moderate growth is observed. ${ }^{46}$ Viability tests indicated that fibroblasts adhered to some extent $(50-60 \%)$ when compared with the adhesion on TCPP and are capable to grow on dextrin hydrogels. In the first stage of the culture, the cells appear round and then attach to the gel surface. As can be seen in Table II, after $1 \mathrm{~h},<30 \%$ of the cells were attached to the hydrogel surface; after $5 \mathrm{~h}$, almost $60 \%$ of the initial cells remained associated to the gels.

As the gels reticulated in the bottom of the wells are slightly opaque, fluorescence labeling was used to facilitate the evaluation of the cell morphology. The results are presented in Figure 6. After $24 \mathrm{~h}$, small aggregates of round cells are visible. After 2 days, larger cell aggregates are visible, and the cells start spreading out from these aggregates. The Live/ Dead assay demonstrates the nontoxic character of the material, because no dead cells are observed.

The CPII evaluation is shown in Figure 7. As can be seen, $48 \mathrm{~h}$ after the cell seeding on the hydrogel surfaces, cell proliferation inhibition reaches about $50 \%$ when compared with control cultures. The direct seeding of the cells onto the hydrogels surfaces resulted in a substantial reduction in the proliferation rate. However, as can be seen by the non-normalized absorbance values [Fig. 6(A-C)], the results show that although with a delay, cells are effectively able to grow, indicating that no deleterious effects are produced by dextrin hydrogels.

\section{CONCLUSIONS}

It was possible to obtain a new class of polymerizable dextrins (dextrin-HEMA) by using a method based on the activation of the hydroxyl groups of HEMA with CDI, followed by their coupling with the polymer, yielding a derivatized material that can be polymerized in aqueous solution to form hydrogels. The comparative study of the stability of dextrin-HEMA/dextrin-VA hydrogels revealed that the firsts are effectively hydrolyzed under physiological conditions. In dextrin-HEMA hydrogels, a progressive swelling is accompanied by a severe mass loss process, which culminates with the complete dissolution of the gels. Accordingly, the rheologic analysis revealed that physical structuring is less pronounced when dextrin is modified with methacrylate side groups. It can be concluded that the dextrin functionalization with HEMA, may be an effective route to modulate the degradation kinetics, tailoring the release profiles to fit the purpose of the controlled delivery. ${ }^{47}$ In light of their biomedical application, the cytotoxicity of the two classes of hydrogels was investigated. The biocompatibility tests, expressed as dysfunction in the metabolic activity of the cells, gave similar results irrespective of the hydrogel type (HEMA and VA), revealing a negligible cell toxicity, allowing cell adhesion and proliferation. These insights into the hydrogel structure-toxicity relationship could be helpful for optimizing the biocompatibility of polymeric delivery systems.

Additionally, the modification of the surface of the hydrogels with RGD peptides, using recombinant starch binding modules (SBM) fused to the bioactive peptides, was proved to significantly improve the cell adhesion and surface spreading (ongoing work). This is a significant advantage on using a starch derived hydrogel (in this case, dextrin), because the availability of SBMs allows a wide variety of bioactive peptides to be driven to the hydrogels surfaces. Furthermore, the interaction of this hydrogel with

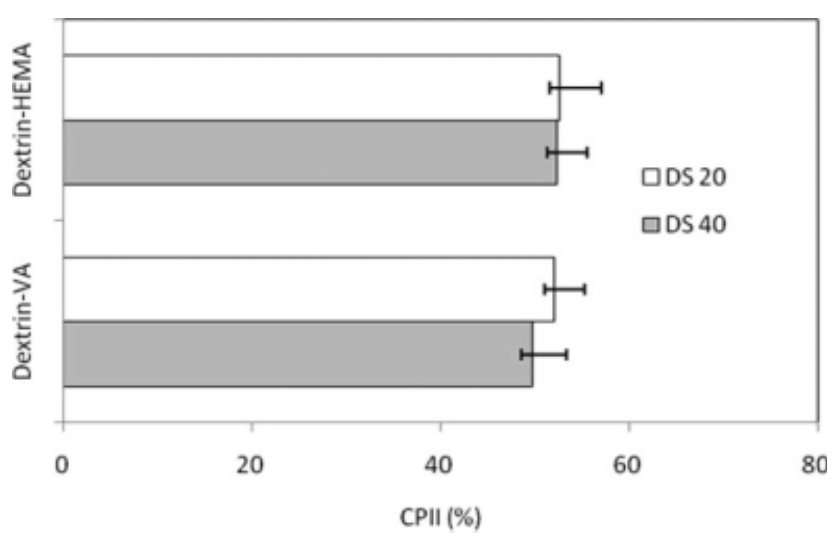

Figure 7. Cell proliferation inhibition index (CPII) (mean $\% \pm$ SD) after $48 \mathrm{~h}$ for different dextrin hydrogel discs. CPII obtained using MTT. Data are from two representative experiments, each one performed in triplicate 
different cell lines and living tissues-subcutaneous implants-is currently being analyzed.

\section{References}

1. Vyavahare N, Kohn J. Photocrosslinked hydrogels based on copolymers of poly(ethylene glycol) and lysine. J Polym Sci Part A: Polym Chem 1994;32:1271-1281.

2. Peppas NA. Biomaterials Science: An Introduction to Materials in Medicine. San Diego: Academic Press; 1996.

3. Kane JB, Tompkins RG, Yarmush ML, Burke JF. Biomaterials Science: An Introduction to Materials in Medicine. San Diego: Academic Press; 1996.

4. McCulloch I, Shalaby SW. Tailored polymeric materials for controlled delivery systems. Washington, DC: American Chemical Society; 1998.

5. Peppas NA, Bures P, Leobandung W, Ichikawa H. Hydrogels in pharmaceutical formulations. Eur J Pharm Biopharm 2000; 50:27-46.

6. Chen TH, Embree HD, Brown EM, Taylor MM, Payne GF. Enzyme-catalyzed gel formation of gelatin and chitosan: Potential for in situ applications. Biomaterials 2003;24:28312841.

7. Drury JL, Mooney DJ. Hydrogels for tissue engineering: Scaffold design variables and applications. Biomaterials 2003;24: 4337-4351.

8. Ishihara M, Ono K, Sato M, Nakanishi K, Saito Y, Yura H, Matsui T, Hattori H, Fujita M, Kikuchi M, Kurita A. Acceleration of wound contraction and healing with a photocrosslinkable chitosan hydrogel. Wound Repair Regen 2001;9:513-521.

9. Okay O, Gurun C. Synthesis and formation mechanism of porous 2-hydroxyethyl methacrylate ethylene-glycol dimethacrylate copolymer beads. J Appl Polym Sci 1992;46:401-410.

10. Walther DH, Sin GH, Blanch HW, Prausnitz JM. Pore-size distributions of cationic 2-hydroxyethyl methacrylate (Hema) hydrogels. Polym Gels Networks 1995;3:29-45.

11. Levy MC, Andry MC. Mixed-walled microcapsules made of cross-linked proteins and polysaccharides-Preparation and properties. J Microencapsul 1991;8:335-347.

12. Schacht E, Bogdanov B, VandenBulcke A, DeRooze N. Hydrogels prepared by crosslinking of gelatin with dextran dialdehyde. React Funct Polym 1997;33:109-116.

13. Disilvio L, Gurav N, Kayser MV, Braden M, Downes S. Biodegradable microspheres-A new delivery system for growth-hormone. Biomaterials 1994;15:931-936.

14. Draye JP, Delaey B, van de Voorde A, van Den Bulcke A, Bogdanov B, Schacht E. In vitro release characteristics of bioactive molecules from dextran dialdehyde cross-linked gelatin hydrogel films. Biomaterials 1998;19:99-107.

15. vanDijkWolthuis WNE, Franssen O, Talsma H, Vansteenbergen MJ, Vandenbosch JJK, Hennink WE. Synthesis, characterization, and polymerization of glycidyl methacrylate derivatized dextran. Macromolecules 1995;28:6317-6322.

16. vanDijkWolthuis WNE, Hoogeboom JAM, vanSteenbergen MJ, Tsang SKY, Hennink WE. Degradation and release behavior of dextran-based hydrogels. Macromolecules 1997; 30:4639-4645.

17. vanDijkWolthuis WNE, KettenesvandenBosch JJ, vanderKerkvanHoof A, Hennink WE. Reaction of dextran with glycidyl methacrylate: An unexpected transesterification. Macromolecules 1997;30:3411-3413.

18. vanDijkWolthuis WNE, Tsang SKY, KettenesvandenBosch JJ, Hennink WE. A new class of polymerizable dextrans with hydrolyzable groups: Hydroxyethyl methacrylated dextran with and without oligolactate spacer. Polymer 1997;38:62356242.
19. vanDijkWolthuis WNE, vanSteenbergen MJ, Underberg WJM, Hennink WE. Degradation kinetics of methacrylated dextrans in aqueous solution. J Pharm Sci 1997;86:413-417.

20. Ferreira L, Carvalho R, Gil MH, Dordick JS. Enzymatic synthesis of inulin-containing hydrogels. Biomacromolecules 2002;3:333-341.

21. Ferreira L, Gil MH, Dordick JS. Enzymatic synthesis of dextran-containing hydrogels. Biomaterials 2002;23:3957-3967.

22. Carvalho J, Goncalves C, Gil AM, Gama FM. Production and characterization of a new dextrin based hydrogel. Eur Polym J 2007;43:3050-3059.

23. Stubbe BG, Braeckmans K, Horkay F, Hennink WE, De Smedt SC, Demeester J. Swelling pressure observations on degrading dex-HEMA hydrogels. Macromolecules 2002;35: 2501-2505.

24. Hansen MB, Nielsen SE, Berg K. Re-examination and further development of a precise and rapid dye method for measuring cell-growth cell kill. J Immunol Methods 1989;119:203210.

25. Behl C, Davis JB, Lesley R, Schubert D. Hydrogen-peroxide mediates amyloid-beta protein toxicity. Cell 1994;77:817827.

26. Berridge MV, Tan AS, McCoy KD, Wang R. The biochemical and cellular basis of cell proliferation assays that use tetrazolium salts. Biochemica 1996;4:15-20.

27. Hern DL, Hubbell JA. Incorporation of adhesion peptides into nonadhesive hydrogels useful for tissue resurfacing. J Biomed Mater Res 1998;39:266-276.

28. Hoffman AS. Hydrogels for biomedical applications. Adv Drug Deliv Rev 2002;54:3-12.

29. Mosmann T. Rapid colorimetric assay for cellular growth and survival-Application to proliferation and cytotoxicity assays. J Immunol Methods 1983;65:55-63.

30. Flory PJ. Principles of Polymer Chemistry. New York: Cornell University Press; 1953.

31. Martens P, Holland T, Anseth KS. Synthesis and characterization of degradable hydrogels formed from acrylate modified poly(vinyl alcohol) macromers. Polymer 2002;43: 6093-6100.

32. Suggs LJ, Kao EY, Palombo LL, Krishnan RS, Widmer MS, Mikos AG. Preparation and characterization of poly(propylene fumarate-co-ethylene glycol) hydrogels. J Biomater Sci: Polym Ed 1998;9:653-666.

33. Suggs LJ, Krishnan RS, Garcia CA, Peter SJ, Anderson JM, Mikos AG. In vitro and in vivo degradation of poly(propylene fumarate-co-ethylene glycol) hydrogels. J Biomed Mater Res 1998;42:312-320.

34. Wong WH, Mooney DJ. Synthesis and properties of biodegradable polymers used as synthetic matrices for tissue engineering. In: Atala A, Mooney DJ, editors. Synthetic Biodegradable Polymer Scaffolds. Boston: Birkhaüser; 1997. p 49-82.

35. Anseth KS, Bowman CN, BrannonPeppas L. Mechanical properties of hydrogels and their experimental determination. Biomaterials 1996;17:1647-1657.

36. Meyvis TKL, De Smedt SC, Demeester J, Hennink WE. Influence of the degradation mechanism of hydrogels on their elastic and swelling properties during degradation. Macromolecules 2000;33:4717-4725.

37. Ferry JD. Viscoelastic Properties of Polymers. New York: John Wiley; 1980.

38. Massia SP, Stark J. Immobilized RGD peptides on surfacegrafted dextran promote biospecific cell attachment. J Biomed Mater Res 2001;56:390-399.

39. Ferreira L, Rafael A, Lamghari M, Barbosa MA, Gil MH, Cabrita AMS, Dordick JS. Biocompatibility of chemoenzymatically derived dextran-acrylate hydrogels. J Biomed Mater Res A 2004;68A:584-596. 
40. Ferreira LS, Cabrita AMS, Rafael A, Gil MH, Dordick JS. Biocompatible and degradable biocatalytic dextran hydrogels. Abstr Papers Am Chem Soc 2003;225:U239-U239.

41. Osterberg E, Bergstrom K, Holmberg K, Schuman TP, Riggs JA, Burns NL, Vanalstine JM, Harris JM. Protein-rejecting ability of surface-bound dextran in end-on and side-on configurationsComparison to PEG. J Biomed Mater Res 1995;29:741-747.

42. Frazier RA, Matthijs G, Davies MC, Roberts CJ, Schacht E, Tendler SJB. Characterization of protein-resistant dextran monolayers. Biomaterials 2000;21:957-966.

43. De Groot CJ, Van Luyn MJA, Van Dijk-Wolthuis WNE, Cadee JA, Plantinga JA, Den Otter W, Hennink WE. In vitro biocompatibility of biodegradable dextran-based hydrogels tested with human fibroblasts. Biomaterials 2001;22:1197-1203.
44. Mann P, Sussmann M, Sarbia M, Meyer-Kirchrath J, Grabitz K, Schror K, Fischer JW. Regulation of hyaluronic acid synthesis in human saphenous vein smooth muscle cells by interleukin-1 and prostaglandins. Naunyn Schmiedebergs Arch Pharmakol 2005;371:R87-R87.

45. Ramamurthi A, Vesely I. Smooth muscle cell adhesion on crosslinked hyaluronan gels. J Biomed Mater Res 2002;60: 196-205.

46. Autissier A, Letourneur D, Le Visage C. Pullulan-based hydrogel for smooth muscle cell culture. J Biomed Mater Res A 2007;82A:336-342.

47. Carvalho J, Coimbra MA, Gama FM. New dextrin-vinylacrylate hydrogel: Studies on protein diffusion and release Carbohydr Polym 2009;75:322-327. 